1 Hacettepe Journal of Mathematics and Statistics

$\bigcap$ Volume 46 (1) (2017), $77-90$

\title{
Functional equivalence of topological spaces and topological modules
}

\author{
Mitrofan M. Choban * and Radu N. Dumbrăveanu ${ }^{\dagger}$
}

\begin{abstract}
Let $R$ be a topological ring and $E, F$ be unitary topological $R$-modules. Denote by $C_{p}(X, E)$ the class of all continuous mappings of $X$ into $E$ in the topology of pointwise convergence. The spaces $X$ and $Y$ are called $l_{p}(E, F)$-equivalent if the topological $R$-modules $C_{p}(X, E)$ and $C_{p}(Y, F)$ are topological isomorphic. Some conditions under which the topological property $\mathcal{P}$ is preserved by the $l_{p}(E, F)$-equivalence (Theorems $6.3,6.4,7.3$ and 8.1 ) are given.
\end{abstract}

Keywords: Function space, topology of pointwise convergence, support, linear homeomorphism, perfect properties, open finite-to-one properties

2000 AMS Classification: Primary 54C35, 54C10, 54C60; Secondary 13F99, $54 \mathrm{C} 40,54 \mathrm{H} 13$

\section{Preliminaries}

Throughout this paper, by a space we will mean a Tychonoff space [9].

A topological semiring is a topological space $R$ equipped with two continuous binary operations $\{+, \cdot\}$, called addition and multiplication, such that (see $[10,11$, $15]):$

1. $(R,+)$ is a topological commutative monoid with identity element 0 and proprieties: $(a+b)+c=a+(b+c), 0+a=a+0=a, a+b=b+a$ for all $a, b, c \in R$.

2. $(R, \cdot)$ is a topological monoid with identity element $1 \neq 0$ and proprieties: $(a \cdot b) \cdot c=a \cdot(\cdot c), 1 \cdot a=a \cdot 1=a, a \cdot b=b \cdot a, a \cdot(b+c)=(a \cdot b)+(a \cdot c), 0 \cdot a=$ 0 for all $a, b, c \in R$.

*Department of Mathematics Tiraspol State University, MD-2069, Chişinău, Moldova, Email : mmchoban@gmail.com

†Department of Mathematics, Bălţl State University, MD-3121, Bălţi, Moldova

Email : rndumbraveanu@gmail.com

Doi : 10.15672/HJMS.2016.402 
Let $G$ be a topological Abelian group under addition operation and $R$ be a topological semiring. We call $G$ a topological $R$-module if on it is defined the continuous operation of multiplication $: R \times G \longrightarrow G$ between an element of $R$ and an element of $G$, say $r a \in G$, where $r \in R$ and $a \in G$, with the following properties: $1 \cdot a=a, 0 \cdot a=0, r(a+b)=r a+r b,(r+s) a=r a+s a, r(s a)=(r s) a$, for any $r, s \in R$ and $a, b \in G$.

Let $R$ be a topological semiring and $E, F$ be topological $R$-modules. The mapping $\varphi: E \rightarrow F$ is a linear mapping if it satisfies the conditions: $\varphi(x+y)=$ $\varphi(x)+\varphi(y)$ and $\varphi(\alpha x)=\alpha \varphi(x)$ for any $x, y \in E$ and $\alpha \in R$.

Fix a space $X$, a topological semiring $R$, and topological $R$-modules $E$ and $F$.

By $C(X, E)$ we will denote the family of all $E$-valued continuous functions with the domain $X$ and by $C_{p}(X, E)$ we will denote the space $C(X, E)$ endowed with the topology of pointwise convergence. Recall that the family of sets of the form $W\left(x_{1}, x_{2}, \ldots, x_{n}, U_{1}, U_{2}, \ldots, U_{n}\right)=\left\{f: C(X, E): f\left(x_{i}\right) \in U_{i}\right.$ for any $\left.i \leq n\right\}$, where $x_{1}, x_{2}, \ldots, x_{n} \in X, U_{1}, U_{2}, \ldots, U_{n}$ are open sets of $E$ and $n \in \mathbb{N}$, is a base of the space $C_{p}(X, E)$.

By $H_{p}(E, F)$ we denote the space of all linear mappings of $E$ into $F$ as a subspace of the space $C_{p}(E, F)$.

The spaces $X$ and $Y$ are called $l_{p}(E, F)$-equivalent if the spaces $C_{p}(X, E)$ and $C_{p}(Y, F)$ are linearly homeomorphic and we denote $X \stackrel{E, F}{\sim} Y$.

A space $X$ is zero-dimensional if $i n d X=0$ (small inductive dimension is zero), i.e., $X$ has a base of clopen (open and closed) subsets.

The following two assertions are evidently.

1.1. Proposition. Fix a topological $R$-module $E$. Then $C_{p}(X, E)$ is a topological $R$-module and $E$ is embedded in a natural way in $C_{p}(X, E)$ as a closed submodule of $C_{p}(X, E)$.

1.2. Proposition. If $E$ is a zero-dimensional topological $R$-module, then $C_{p}(X, E)$ is a zero-dimensional topological $R$-module too.

\section{The evaluation mapping}

Let $X$ be a space, $R$ be a topological semiring and $E$ be a non-trivial topological $R$-module. Fix $x \in X$. Then the mapping $\xi_{x}: C_{p}(X, E) \rightarrow E$ defined by $\xi_{x}(f)=$ $f(x)$ is called the evaluation mapping at $x$ (see, by instance, [1]).

We now define the canonical evaluation mapping $e_{X}: X \rightarrow C_{p}\left(C_{p}(X, E), E\right)$, where $e_{X}(x)=\xi_{x}$ for any $x \in X$.

The proofs of the following two assertions are standard (see [8]).

2.1. Proposition. The evaluation mapping $\xi_{x}: C_{p}(X, E) \rightarrow E$ is continuous and linear for every point $x \in X$.

2.2. Proposition. The canonical evaluation mapping $e_{X}: X \rightarrow C_{p}\left(C_{p}(X, E), E\right)$ is continuous. Moreover, the set $e_{X}(X)$ is closed in the space $C_{p}\left(C_{p}(X, E), E\right)$.

Let $X$ and $Y$ be spaces, $\Phi$ be a family of functions $f: X \rightarrow Y$. We say that $\Phi$ separates points of $X$ (or simply is separating [1]) if for any $x, y \in X, x \neq y$, there exists $f \in \Phi$ such that $f(x) \neq f(y)$. We also say that $\Phi$ separates points from closed sets (or is regular [1]) if for any non-empty closed subset $B$ of $X$, any point 
$x \in X \backslash B$ and any two points $a, b \in Y$ there exists $f \in \Phi$ such that $f(x)=a$ and $f(B)=b$.

2.3. Proposition. If $C_{p}(X, E)$ is a regular family, then the canonical evaluation mapping $e_{X}: X \rightarrow C_{p}\left(C_{p}(X, E), E\right)$ is a homeomorphism from $X$ to the closed subspace $e_{X}(X)$ of $C_{p}\left(C_{p}(X, E), E\right)$.

A space $X$ is called $R$-Tychonoff if for any closed subset $B$ of $X$, any point $a \in X \backslash F$ there exists $g \in C(X, R)$ such that $g(a)=1$ and $B \subseteq g^{-1}(0)$.

The product of $R$-Tychonoff spaces is an $R$-Tychonoff space. The subspace of an $R$-Tychonoff is an $R$-Tychonoff space.

Remark. Let $X$ be an $R$-Tychonoff space and $E$ be a non-trivial topological $R$ module. Then $X$ is a Tychonoff space, and for each closed set $B$ of $X$, any point $a \in X \backslash B$ and any points $b, c \in E$ there exists $f \in C(X, E)$ such that $f(a)=b$ and $f(B)=c$.

The proofs of the following two assertion is elementary.

2.4. Proposition. If $i n d X=0$, then the space $X$ is $R$-Tychonoff.

Let $R$ be a topological semiring. A topological $R$-module $E$ is called:

(i) simple if it does not contain a non-trivial submodule over $R$.

(ii) locally simple if $E$ is not trivial and there exists an open subset $U$ of $E$ such that $0 \in U$ and $U$ do not contains non-trivial $R$-submodules of $E$.

2.5. Example. If $R$ is a field, then $R$ is a simple topological $R$-module. Let $\mathbb{R}$ be the field of real numbers and $\mathbb{K}$ be the field of complex numbers. Then $\mathbb{K}$ is locally simple and not simple $\mathbb{R}$-module.

We mention the following obvious fact.

2.1. Lemma. Let $R$ be a topological semiring and $E$ be an $R$-module. Then $R a$ is an R-submodule for any $a \in E$.

Fix a space $X$ and two topological $R$-modules $E$ and $F$. We define $M_{p}(X, E, F)$ $=H_{p}\left(C_{p}(X, E), F\right)$ the subspace of all linear mappings from $C_{p}(X, E)$ into $F$. Let $M_{p}(X, E)=M_{p}(X, E, E)$. Now we define $L_{p}(X, E) \subseteq C_{p}\left(C_{p}(X, E), E\right)$ as follows $L_{p}(X, E)=\left\{\alpha_{1} x_{1}+\alpha_{2} x_{2}+\ldots+\alpha_{n} x_{n}: \alpha_{i} \in R, x_{i} \in e_{X}(X), i \leq n \in \mathbb{N}\right\}$.

By construction, we have $L_{p}(X, E) \subseteq M_{p}(X, E)$. As a rule $L_{p}(X, E) \neq M_{p}(X, E)$ (see [8]).

2.6. Proposition. Let $R$ be a topological semiring, $E$ be a topological $R$-module and $X$ be a space. Then for any $g \in C(X, E)$ there exists a unique linear mapping $\bar{g} \in H_{p}\left(L_{p}(X, E), E\right)$ such that $g=\bar{g} \circ e_{X}$, where $e_{X}: X \rightarrow L_{p}(X, E)$ is the evaluation mapping.

Proof. Let $E_{f}=E$ for any $f \in C_{p}(X, E)$. By definition, $e_{X}(X) \subseteq L_{p}(X, E) \subseteq$ $E^{C(X, E)}=\Pi\left\{E_{f}: f \in C(X, E)\right\}$. We have $e_{X}(x)=\xi_{x}$ for any $x \in X$ and $L_{p}(X, E)$ is the submodule of $E^{C(X, E)}$ generated by the set $e_{X}(X)$. We consider the projection $\pi_{f}: E^{C(X, E)} \longrightarrow E_{f}=E$. Let $\bar{f}=\pi_{f} \mid L_{p}(X, E): L_{p}(X, E) \longrightarrow E$ is the desired linear mapping. 
2.7. Theorem. Let $R$ be a semiring, $E$ be a topological $R$-module and $X$ be a space. Consider the space $e_{X}(X)$, where $e_{X}: X \rightarrow L_{p}(X, E)$ is the evaluation mapping. Then the topological $R$-modules $C_{p}(X, E), C_{p}\left(e_{X}(X), E\right)$ and $H_{p}\left(L_{p}(X, E), E\right)$ are linearly homeomorphic.

Proof. Let $E_{f}$ for any $f \in C_{p}(X, E)$. By definition, $e_{X}(X) \subseteq L_{p}(X, E) \subseteq$ $E^{C_{p}(X, E)}=\Pi\left\{E_{f}: f \in C(X, E)\right\}$. We consider the projection $\pi_{f}: E^{C(X, E)} \longrightarrow$ $E_{f}=E$. Let $\bar{f}=\pi_{f} \mid L_{p}(X, E): L_{p}(X, E) \longrightarrow E$. Then $\bar{f}$ and $\pi_{f}$ are continuous linear mappings.

If $g: e_{X}(X) \rightarrow E$ is a continuous mapping, then $g \circ e_{X}=f$ for a unique $f \in C(X, E)$. Therefore, $g=\pi_{f} \mid e_{X}(X)$ and the correspondence $f \rightarrow \pi_{f} \mid e_{X}(X)$ is a linear homeomorphism of $C_{p}(X, E)$ onto $C_{p}\left(e_{X}(X), E\right)$.

Hence, without loss of generality, we can assume that $X=e_{X}(X) \subseteq L_{p}(X, E)$.

By virtue of Proposition 2.6, the correspondence

$\psi: C_{p}(X, E) \longrightarrow H_{p}\left(L_{p}(X, E), E\right)$, where $\psi(f)=\bar{f}$, is a one-to-one linear mapping of $C(X, E)$ onto $H_{p}\left(L_{p}(X, E), E\right)$.

For each $y \in L_{p}(X, E)$ there exist the minimal $n=n(y) \in \mathbb{N}$, the unique points $x_{1}(y), \ldots, x_{n}(y) \in X$ and the unique points $\alpha_{1}(y), \ldots, \alpha_{n}(y) \in R$ such that $y=\alpha_{1}(y) x_{1}(y)+\ldots+\alpha_{n}(y) x_{n}(y)$. Hence, the correspondence $\psi$ is continuous and linear. Since $\psi(f) \mid X=f$, the mapping $\psi^{-1}$ is continuous.

2.8. Corollary. Let $X, Y$ be spaces and $R$ be a locally simple $R$-module. The spaces $C_{p}(X, R)$ and $C_{p}(Y, R)$ are linearly homeomorphic if and only if the spaces $L_{p}(X, R)$ and $L_{p}(Y, R)$ are linearly homeomorphic.

2.2. Lemma. Let $X$ be an $R$-Tychonoff space, $Z$ be a closed subspace of $X, E$ be a topological $R$-module and $g: X \longrightarrow E$ be a continuous mapping. For any finite subset $B$ of $X \backslash Z$ and any function $f: B \longrightarrow E$ there exists a continuous function $\varphi: X \longrightarrow E$ such that $f=\varphi \mid B$ and $\varphi|Z=g| Z$.

Proof. Fix a family $\left\{U_{x}: x \in B\right\}$ of open subsets of $X$ such that $x \in U_{x} \subseteq X \backslash Z$ for each $x \in B$ and $U_{x} \cap U_{y}=\varnothing$ for each distinct points $x, y \in B$. For each $x \in B$ fix a continuous function $f_{x}: X \longrightarrow E$ such that $f_{x}(x)=f(x)-g(x)$ and $f_{x}\left(X \backslash U_{x}\right)=0$. Let $f_{B}(y)=\sum\left\{f_{x}(y): x \in B\right\}$. By construction, the function $f_{B}$ is continuous, $f_{B}(Z)=0$ and $f_{B}(x)=f(x)-g(x)$ for each $x \in B$. Obviously, $\varphi$ $=f_{B}+g$ is the desired function.

For any subspace $Y$ of a space $X$ we put $C_{p}(Y \mid X, E)=\{f \mid Y: f \in C(X, E)\}$. A subspace $Y$ of $X$ is $E$-full if $C(Y \mid X, E)=C(Y, E)$.

A space $X$ is called compactly E-full if $C(Y \mid X, E)=C(Y, E)$ for any compact subspace $Y$ of $X$.

The following assertion is well-known (see [8]).

2.3. Lemma. Let $X$ be a zero-dimensional space and $E$ be a metrizable space. Then $X$ is a compactly $E$-full space. Moreover, for any compact subset $Y$ of $X$ and any $f \in C(Y, E)$ there exists $g \in C(X, E)$ such that $g(X) \subseteq f(Y)$ and $f=\left.g\right|_{Y}$.

\section{The support mapping}

Fix a topological semiring $R$ and non-trivial topological $R$-modules $E$ and $F$. 
Consider a space $X$ and a functional $\mu \in M_{p}(X, E, F)$. We put $\mathcal{S}(\mu)=\{B \subseteq X$ : if $B \subseteq f^{-1}(0)$, then $\left.\mu(f)=0\right\}$. Obviously, $X \in \mathcal{S}(\mu)$. Thus the set $\mathcal{S}(\mu)$ is nonempty.

The set $\operatorname{supp}_{X}(\mu)$ is the family of all points $x \in X$ such that for each neighbourhood $U$ of $x$ in $X$ there exists $f \in C_{p}(X, E)$ such that $f(X \backslash U)=0$ and $\mu(f) \neq 0$ (see $[2,12]$, for $E=R=\mathbb{R},[3,14]$ for $R=\mathbb{R},[8]$ when $R$ is a topological ring).

If $f \in C_{p}(X, E)$ and $U$ is an open neighbourhood of 0 in $E$, then we put $A(f, L, U)=\left\{g \in C_{p}(X, E): f(x)-g(x) \in U\right.$ for any $\left.x \in L\right\}$. The family $\left\{A(f, L, U): f \in C_{p}(X, E), L\right.$ is finite subset of $X, U$ is open neighbourhood of 0 in $E\}$ is an open base of the space $C_{p}(X, E)$.

3.1. Theorem. Let $X$ be a $R$-Tychonoff space, $E$ and $F$ be non-trivial topological $R$-modules, $\mu \in M_{p}(X, E, F)$ and $\mu \neq 0$. If $F$ is a locally simple topological $R$ module, then:

1. There exists a finite set $K \in \mathcal{S}(\mu)$ such that $\operatorname{supp}_{X}(\mu) \subseteq K$.

2. $\operatorname{supp}_{X}(\mu) \in \mathcal{S}(\mu)$ and $\operatorname{supp}_{X}(\mu)$ is a finite non-empty subset of $X$.

3. $\operatorname{supp}_{X}(\mu)=\cap \mathcal{S}(\mu)$.

Proof. Fix an open subset $U_{0}$ of $C_{p}(X, E)$ such that $0 \in U_{0}$ and an open subset $W_{0}$ of $F$ such that $0 \in W_{0}, W_{0}$ do not contains non-trivial $R$-submodules of $F$ and $\mu\left(U_{0}\right) \subseteq W_{0}$.

There exist a finite subset $K$ of $X$ and an open subset $V_{0}$ of $E$ such that $0 \in V_{0}$ and $0 \in A\left(0, K, V_{0}\right) \subseteq U_{0}$. Hence $\mu(f) \in W_{0}$ for each $f \in A\left(0, K, V_{0}\right)$.

Let $f \in C_{p}(X, E)$ and $f(K)=0$. Then $\alpha f \in A\left(0, K, V_{0}\right)$ for each $\alpha \in R$. Hence $\mu(\alpha f) \in W_{0}$ for each $\alpha \in R$. Thus $R \cdot \mu(f) \subseteq V_{0}$ and $R \cdot \mu(f)$ is the trivial $R$-submodule. Thus $\mu(f)=0$ and $K \in \mathcal{S}(\mu)$. In this case $\operatorname{supp}_{X}(\mu) \subseteq K$. Hence $\operatorname{supp}_{X}(\mu)$ is a finite set and $K$ is a finite set from $\mathcal{S}(\mu)$.

Let $L \in \mathcal{S}(\mu)$ be a finite set and $x_{0} \in L \backslash \operatorname{supp}_{X}(\mu)$. Then $L_{1}=L \backslash\left\{x_{0}\right\} \in \mathcal{S}(\mu)$. Really, since $x_{0} \notin \operatorname{supp}_{X}(\mu)$, there exists an open subset $H$ of $X$ such that $x_{0} \in H$ and $\mu(f)=0$ provided $f(X \backslash H)=0$. We can assume that $H \cap L=\left\{x_{0}\right\}$. Let $f \in C_{p}(X, E)$ and $f\left(L_{1}\right)=0$. There exists $h \in C(X, E)$ such that $h\left(x_{0}\right)=f\left(x_{0}\right)$ and $h(X \backslash H)=0$. We put $g(x)=f(x)-h(x)$ for any $x \in X$. Since $h(X \backslash H)=0$, we have $\mu(h)=0$. By construction, $g(L)=0$ and $\mu(g)=0$. Hence $f=g+h$ and $\mu(f)=\mu(g+h)=\mu(g)+\mu(h)=0$. Hence $L_{1} \in \mathcal{S}(\mu)$. Since $K \in \mathcal{S}(\mu)$ and $K \backslash \operatorname{supp}_{X}(\mu)$ is a finite set, we have $\operatorname{supp}_{X}(\mu) \in \mathcal{S}(\mu)$. In particular, we have $\operatorname{supp}_{X}(\mu)=\cap \mathcal{S}(\mu)$.

The following assertions are obviously:

3.2. Proposition. Let $n \geq 1, x_{1}, x_{2}, \ldots, x_{n}$ are distinct points of $X, \alpha_{1}, \alpha_{2}, \ldots, \alpha_{n} \in$ $R$ and $\mu(f)=\Sigma\left\{\alpha_{i} f\left(x_{i}\right): i \leq n\right\}$ for each for each $f \in C_{p}(X, E)$, then:

1. $\mu \in L_{p}(X, E)$ and $\operatorname{supp}_{X}(\mu) \subseteq\left\{x_{1}, x_{2}, \ldots, x_{n}\right\}$.

2. If for each $i \leq n$ the set $\alpha_{i} E$ is a non-trivial $R$-submodule of $E$, then $\operatorname{supp}_{X}(\mu)=\left\{x_{1}, x_{2}, \ldots, x_{n}\right\}$.

\section{Topological properties of the mapping $s u p p_{X}$}

Fix a topological semiring $R$. Let $X$ be a space, $E$ and $F$ be two non-trivial topological $R$-modules. 
Recall that a set-valued mapping $f: X \rightarrow 2^{Y}$ is lower semicontinuous (l.s.c) if for every open subset $U$ of $Y$ the inverse image of $U, f^{-1}(U)=\{x \in X: f(x) \cap U \neq$ $\varnothing\}$ is open in $X$.

The correspondence $\operatorname{supp}_{X}$ is a set-valued mapping of the space $M_{p}(X, E, F)$ into $X$. For $H \subseteq M_{p}(X, E, F)$ we put $\operatorname{supp}_{X}(H)=\cup\left\{\operatorname{supp}_{X}(\mu): \mu \in H\right\}$.

4.1. Proposition. If $F$ is a locale simple $R$-module, then the set-valued mapping $\operatorname{supp}_{X}: M_{p}(X, E, F) \rightarrow X$ is 1.s.c.

Proof. We follow very closely the proof of [3, Property 4.2] and [12, Lemma 6.8.2 (4)].

Let $U$ be an open subset of $X$, and put $V=\operatorname{supp}_{X}^{-1}(U)$, i.e., $V=\{\mu \in$ $\left.M_{p}(X, E, F): \operatorname{supp}_{X}(\mu) \cap U \neq \varnothing\right\}$. Let $\mu \in V$, and take $x_{0} \in \operatorname{supp}_{X}(\mu) \cap U$. Fix an open subset $W$ of $X$ such that $x_{0} \in W \subseteq c l_{X} W \subseteq U$. Then there exists $f \in C(X, E)$ such that $f(X \backslash W)=\{0\}$ and $\mu(f) \neq 0$. Let $H=\left\{\eta \in M_{p}(X, E, F)\right.$ : $\eta(f) \neq 0\}$. Since the set $\{0\}$ is closed in $F, H$ is the basic open set $W(f, F \backslash\{0\})$ $=\left\{\eta \in M_{p}(X, E, F): \eta(f) \in F \backslash\{0\}\right\}$ and $\mu \in W(f, F \backslash\{0\})$.

We affirm that $H \subseteq V$. By contradiction, suppose that $\eta \in H \backslash V$, i.e. $\eta(f) \neq 0$ and $\operatorname{supp}_{X}(\eta) \cap U=\varnothing$. Then $X \backslash c l_{X} W$ is an open neighbourhood of $\operatorname{supp}_{X}(\eta)$ and, since $f\left(X \backslash c l_{X} W\right)=\{0\}$, applying Theorem 3.1, we get that $\eta(f)=0$. A contradiction, hence $V$ is open in $M_{p}(X, E, F)$.

A subset $L$ of a space $X$ is bounded if any continuous real-valued function $f: X \longrightarrow \mathbb{R}$ is bounded on $L$.

A subset $L$ of a topological $R$-module $E$ is called:

(i) precompact or totally $a$-bounded if for any neighbourhood $U$ of 0 in $E$ there exists a finite subset $A$ of $E$ such that $L \subseteq A+U=U+A$;

(ii) $a$-bounded if for any neighbourhood $U$ of the 0 in $E$ there exists $n \in \mathbb{N}$ such that $L \subseteq n U$.

Any bounded set is precompact. In a topological vector space over field of reals any precompact set is $a$-bounded.

A topological $R$-module $E$ is called locally bounded if there exists an $a$-bounded neighbourhood $U$ of 0 in $E$ such that $E=\cup\{n U: n \in \mathbb{N}\}$ and for any $a \in E$, $a \neq 0$, and any $n \in \mathbb{N}$ there exists $t \in R$ such that $t a \notin n U$. In this case the set $U$ does not contain $R$-submodules of $E$ and $E$ is a locally simple $R$-module.

4.2. Example. Let $E$ be a normed vector space over reals $\mathbb{R}$. Then $E$ is a locally bounded $\mathbb{R}$-module.

4.3. Example. Let $E$ be a topological vector space over reals $\mathbb{R}$ and there exists a number $q>0$ and a functional $\|\|:. E \longrightarrow \mathbb{R}$ such that:

1. $0<q \leq 1$.

2. $\|x\| \geq 0$ for any $x \in E$.

3. If $\|x\|=0$, then $x=0$.

4. $\|x+y\| \leq\|x\|+\|y\|$ for all $x, y \in E$.

5. $\|\lambda x\| \leq|\lambda|^{q}|| x||$ for all $x \in E$ and $\lambda \in \mathbb{R}$.

6. If $x \neq 0$ then $\lim _{\lambda \rightarrow+\infty}\|\lambda x\|=+\infty$.

The functional $\|$.$\| is called a q$-norm, if the family $\{V(0, r)=\{x:\|x\|<r\}$ : $r>0\}$ is a base of $E$ at 0 . Any $q$-normed space is locally bounded. 
4.4. Theorem. Let $F$ be a locally bounded topological $R$-module, $B$ be a submodule of $F$ and $X$ be an $R$-Tychonoff space with the following properties:

(b) : for any non-bounded subset $L$ of $X$ there exists $f \in C(X, B)$ such that the set $f(L)$ is not $a$-bounded in $F$;

$(r): B$ is topological isomorphic to some $R$-submodule of $E$.

Then:

(i) The set $\operatorname{supp}_{X}(H)$ is bounded in $X$ for any $a$-bounded subset $H$ of $M_{p}(X, E, F)$.

(ii) The set $\operatorname{supp}_{X}(H)$ is bounded in $X$ for any totally $a$-bounded subset $H$ of $M_{p}(X, E, F)$.

(iii) The set $\operatorname{supp}_{X}(H)$ is bounded in $X$ for any bounded subset $H$ of $M_{p}(X, E, F)$.

Proof. We can assume that $B \subseteq E$ too. Since $B$ is a non $a$-bounded subset of $F$ there exists an open subset $W_{0}$ of $F$ such that $0 \in W_{0}$ and $B \backslash n W_{0} \neq \varnothing$ for each $n \in \mathbb{N}$. Moreover, If $H \subseteq B$ is a non $a$-bounded of $F$ then $H$ is a non $a$-bounded of $B$ too.

Since $F$ is locally bounded we can fix an open neighbourhood $W_{1}$ of 0 in $E$ such that the set $W_{1}$ is $a$-bounded, $F=\bigcup\left\{n W_{1}: n \in \mathbb{N}\right\}$ and for any $a \in F, a \neq 0$, and for any $n \in \mathbb{N}$ there exists $t \in R$ such that $t a \notin n W_{1}$.

Now fix two open neighbourhoods $W_{2}$ and $W_{3}$ of 0 in $F$ such that $W_{2}=-W_{2} \subset$ $3 W_{2}=W_{2}+W_{2}+W_{2} \subseteq W_{3}=-W_{3} \subseteq W_{1} \cap W_{0}$.

By construction, $W_{1} \subseteq k W_{2}$ for some $k \in \mathbb{N}$.

Hence the sets $W_{2}$ and $W_{3}$ have the following properties:

- $W_{2}$ and $W_{3}$ are $a$-bounded subsets of $E$;

- $F=\bigcup\left\{n W_{2}: n \in \mathbb{N}\right\}=\bigcup\left\{n W_{3}: n \in \mathbb{N}\right\}$;

- if $L$ is a bounded or a precompact subset of $F$, then $L \subseteq n W_{2}$ for some $n \in \mathbb{N}$;

- if $a \in F, a \neq 0$, then for any $n \in \mathbb{N}$ there exists $t \in R$ such that $t a \notin n W_{3}$.

Since $B$ is a non $a$-bounded subset of $F$ and $W_{3}$ is an $a$-bounded of $F$, we have $B \backslash n W_{3} \neq \varnothing$ for each $n \in \mathbb{N}$.

If $\mu \in M_{p}(X, E, F)$ and $\mu \neq 0$, then $\operatorname{supp}_{X}(\mu)$ is a finite non-empty subset of $X$.

We can assume that $C(X, B) \subseteq C(X, E)$ and $C(X, B) \subseteq C(X, F)$.

Suppose that the set $H$ is $a$-bounded or precompact in $M_{p}(X, E, F)$ and the set $\operatorname{supp}_{X}(H)$ is not bounded in $X$. Fix $f \in C(X, B)$ such that the set $f\left(\operatorname{supp}_{X}(H)\right)$ is not $a$-bounded in $F$.

By induction, we shell construct a sequence $\left\{\mu_{n}: n \in \mathbb{N}\right\} \subseteq H$, a sequence $\left\{U_{k}: k \in \mathbb{N}\right\}$ of open subsets of $X$, a sequence $\left\{x_{n} \in \operatorname{supp}_{X}\left(\mu_{n}\right): n \in \mathbb{N}\right\}$ and a sequence $\left\{h_{k} \in C(X, B): n \in \mathbb{N}\right\}$ with properties:

1. $x_{i} \in U_{i}, h_{i}\left(X \backslash U_{i}\right)=0$ for any $i \in \mathbb{N}$;

2. $\left\{U_{n}: n \in \mathbb{N}\right\}$ is a discrete family of subsets of $X$;

3. $\mu_{n}\left(h_{n}\right) \notin n W$;

4. $\operatorname{supp}_{X}\left\{\mu_{1}, \mu_{2}, \ldots, \mu_{n}\right\} \cap c l_{X} U_{n+1}=\varnothing$;

5. $f\left(U_{n}\right) \subseteq f\left(x_{n}\right)+W_{0}$ and $f\left(x_{n+1}\right) \notin \bigcup\left\{f\left(x_{i}\right)+W: i \leq n\right\}$ for each $n \in \mathbb{N}$;

Fix $\mu_{1} \in H$ and $x_{1} \in \operatorname{supp}_{X}\left(\mu_{1}\right)$. There exists an open subset $U_{1}$ of $X$ and $g_{1} \in C(X, B)$ such that $f\left(U_{1}\right) \subseteq W_{0}+f\left(x_{1}\right), g_{1}\left(X \backslash U_{1}\right)=0$ and $\mu_{1}\left(g_{1}\right) \neq 0$. There exists $\alpha_{1} \in R$ such that $\alpha_{1} \mu_{1}(g) \notin W_{3}$. We put $h_{1}=\alpha_{1} g_{1}$.

Assume that $n \geq 1$ and the objects $\left\{h_{i}, x_{i}, U_{i}, \mu_{i}: i \leq n\right\}$ are constructed. We put $M_{n}=\bigcup\left\{\operatorname{supp}_{X}\left(\mu_{i}\right): i \leq n\right\}$. The set $M_{n}$ is finite. Hence the set 
$f\left(\operatorname{supp}_{X}(H)\right) \backslash f\left(M_{n}\right)$ is not $a$-bounded in $F$. For some $m_{n} \in \mathbb{N}$ we have $f\left(M_{n}\right) \subseteq$ $m_{n} W_{0}$.

Fix $\mu_{n+1} \in H$ and $x_{n+1} \in \operatorname{supp}_{X}(H)$ such that $f\left(x_{n+1}\right) \in B \backslash m_{n} W$. There exists an open subset $U_{n+1}$ of $X$ and $g_{n+1} \in C(X, B)$ such that $x_{n+1} \in U_{n+1}$, $f\left(U_{n+1}\right) \subseteq f\left(x_{n+1}\right)+W_{0}, g_{n+1}\left(X \backslash U_{n+1}\right)=0, c l_{X} U_{n+1} \cap M_{n}=\varnothing$ and $M_{n+1}\left(g_{n+1}\right) \neq$ 0 . There exists $\alpha_{n+1} \in R$ such that $\alpha_{n+1} \mu_{n+1}\left(g_{n+1}\right) \notin(n+1) W$. We put $h_{n+1}=\alpha_{n+1} g_{n+1}$. That completes the inductive construction. The objects $\left\{x_{m}, \mu_{n}, h_{n}, U_{n}\right\}$ are constructed for all $n \in \mathbb{N}$. Let $h=\Sigma\left\{h_{n}: n \in \mathbb{N}\right\}$. Since $\left\{U_{n}: n \in \mathbb{N}\right\}$ is a discrete family and $h_{n}\left(X \backslash U_{n}\right)=0$ for any $n \in \mathbb{N}$, we have $h \in C(X, B)$. By construction, $\mu_{n}(h)=\mu_{n}\left(h_{n}\right) \notin n W_{0}$ for any $n$. Then $\left\{\mu_{n}(h): n \in \mathbb{N}\right\}$ is a not $a$-bounded subset of $E$. Since the set $H$ is $a$-bounded, the set $\{\mu(h): \mu \in H\}$ is $a$-bounded too, a contradiction. The proof is complete.

Remark. Any normed space is a locally bounded $\mathbb{R}$-module. If $E$ is a non-trivial normed space, then for any non-bounded subset $L$ of the space $X$ there exists $f \in C(X, E)$ such that the set $f(L)$ is not bounded in $E$. For a normed space $E$ Theorem 4.4 was proved by V. Valov in [14]. For a ring $R$ and $E=F$ Theorem 4.4 was proved in [8].

A space $X$ is $\mu$-complete if any closed bounded subset of $X$ is compact.

A space $X$ is Dieudonne complete if the maximal uniformity on $X$ is complete. Any Dieudonné complete space is $\mu$-complete.

Denote by $P X$ the space $X$ with the $G_{\delta}$-topology generated by the $G_{\delta}$-subsets of $X$. The set $\delta-c l_{X} H=c l_{P X} H$ is called the $G_{\delta}$-closure of the set $H$ in $X$. If $\delta-c l_{X} H=H$, then we say the set $H$ is $G_{\delta}$-closed.

If the space $X$ is $\mu$-complete, then any $G_{\delta}$-closed subspace of $X$ is $\mu$-complete.

A tightness of a space $X$ is the minimal cardinal number $\tau$ for which for any subset $L \subseteq X$ and any point $x \in c l_{X} L$ there exists a subset $L_{1} \subseteq L$ such that $\left|L_{1}\right| \leq \tau$ and $x \in c l_{X} L_{1}$.

We denote by $t(X)$ and $l(X)$ the tightness and the Lindelöf numbers respectively of a space $X$.

The following four propositions were proved in [8] (see [1] for $E=\mathbb{R}$ ).

4.5. Proposition. Assume that $E$ is a metrizable and $l\left(X^{n}\right) \leq \tau$ for any $n \in \mathbb{N}$. Then $t\left(C_{p}(X, E)\right) \leq \tau$.

4.6. Proposition. Let $X$ and $E$ be spaces and $t(X) \leq \aleph_{0}$. Then $C_{p}(X, E)$ is a $G_{\delta}$-closed subspace of the space $E^{X}$. Moreover, if $E$ is $\mu$-complete then the space $C_{p}(X, E)$ is $\mu$-complete too.

4.7. Proposition. Let $F$ and $E$ be topological $R$-modules and $H_{p}(F, E)$ be the space of all linear continuous mappings of $F$ into $E$. Then $H_{p}(F, E)$ is a closed subspace of the space $C_{p}(F, E)$.

4.8. Corollary. Let $E$ and $F$ be topological $R$-modules and $t(F) \leq \aleph_{0}$. Then $H_{p}(F, E)$ is a $G_{\delta}$-closed subset of $E^{F}$. In particular, if $E$ is $\mu$-complete, then space $H_{p}(F, E)$ is $\mu$-complete too.

4.9. Proposition. Let $Y$ be a subspace of the space $X, E$ be a non-trivial topological $R$-module, $X$ be an $R$-Tychonoff space and $p_{Y}(f)=\left.f\right|_{Y}$ for each 
$f \in C_{p}(X, E)$. Then the mapping $p_{Y}: C_{p}(X, E) \longrightarrow C_{p}(Y \mid X, E)$ has the following properties:

(i) $p_{Y}$ is a continuous mapping.

(ii) If the set $Y$ is closed in $X$, then the mapping $p_{Y}$ is open.

(iii) If $Y$ is dense in $X$, then $p_{Y}$ is a one-to-one correspondence.

(iv) The subspace $C_{p}(Y \mid X, E)$ is dense in the $C_{p}(Y, E)$.

4.10. Theorem. Let $E$ be a metrizable $R$-module, $F$ be a locally bounded metrizable $R$-module, $B$ be a closed submodule of $F$ and $X$ be an $R$-Tychonoff space with the following properties:

(b) : for any non-bounded subset $L$ of $X$ there exists $f \in C(X, B)$ such that the set $f(L)$ is not $a$-bounded in $F$;

$(r): B$ is topological isomorphic to some $R$-submodule of $E$;

(c) : $X$ be an $R$-Tychonoff compactly $E$-full space.

Then the space $X$ is $\mu$-complete if and only if the space $M_{p}(X, E, F)$ is $\mu$ complete.

Proof. By virtue of Proposition 2.3, we can assume that $X=e_{X}(X)$ is a subspace of the space $M_{p}(X, E, B)$. From Proposition 2.2 it follows that the subspace $X$ is closed in $M_{p}(X, E, B)$. Obviously, $M_{p}(X, E, B)$ is a closed subspace of the space $M_{p}(X, E, F)$.

Let $M_{p}(X, E, F)$ be a $\mu$-complete space. Since $X$ is a closed subspaces of $M_{p}(X, E, B)$ and $M_{p}(X, E, F)$, the space $X$ is $\mu$-complete too.

Assume that $X$ is a $\mu$-complete space. Let $\Phi$ be a closed bounded subset of $M_{p}(X, E, F)$. Then the closure $Y$ of the set $\cup\left\{\operatorname{supp}_{X}(\mu): \mu \in \Phi\right\}$ is a compact subset of $X$.

The restriction mapping $p_{Y}: C_{p}(X, E) \longrightarrow C_{p}(Y, E)$ is an open continuous linear mapping of the $R$-module $C_{p}(X, E)$ onto the $R$-module $C_{p}(Y, E)$.

Claim 1. The dual mapping $\varphi: F^{C(Y, E)} \longrightarrow F^{C(X, E)}$ is a linear embedding and the set $\varphi\left(F^{C(Y, E)}\right)$ is closed in $F^{C(X, E)}$.

The proof of this fact is similar with the prof of Proposition 0.4.6 from [1].

By construction, we have $\Phi \subseteq \varphi\left(M_{p}(Y, E, F)\right) \subseteq M_{p}(X, E, F)$.

Claim 2. $\varphi\left(M_{p}(Y, E, F)\right)$ is a closed subset of the subspaces $M_{p}(X, E, F)$ and $C_{p}\left(C_{p}(X, E), E\right)$ of the space $E^{C(X, E)}$.

Follows from Claim 1 and Proposition 4.7.

Claim 3. $\varphi\left(C_{p}\left(C_{p}(Y, E), F\right)\right) \subseteq C_{p}\left(C_{p}(X, E), F\right)$.

Follows from the continuity of the mapping $p_{Y}$.

Claim 4. The sets $\varphi\left(M_{p}(X, E, F)\right)$ and $\varphi\left(C_{p}\left(C_{p}(Y, E), F\right)\right)$ are $G_{\delta^{-}}$closed in $F^{C(X, E)}$.

Since $Y$ is compact, from Proposition 4.5 it follows that $t\left(C_{p}(Y, E)\right)=\aleph_{0}$. Then, from Proposition 4.6 it follows that $C_{p}\left(C_{p}(Y, E), F\right)$ is a $G_{\delta}$-closed subset of the space $F^{C(Y, E)}$. From Claim 1 it follows that $\varphi\left(C_{p}\left(C_{p}(Y, E), F\right)\right)$ is $G_{\delta}$-closed in $F^{C(X, E)}$. Corollary 4.8 completes the proof of the claim.

Let $G$ be the $G_{\delta}$-closure of the set $\left.C_{p}\left(C_{p}(X, E), E\right)\right)$ in $E^{C(X, E)}$. We have $M_{p}(X, E, F) \subseteq G$. Hence $\Phi$ is a bounded subset of the space $G$.

Claim 5. The sets $\varphi\left(M_{p}(X, E, F)\right)$ and $\varphi\left(C_{p}\left(C_{p}(Y, E), F\right)\right)$ are closed in $G$.

Follows from Claim 4. 
Since $F$ is a metrizable space, $F$ is a $\mu$-complete space. Thus $\Phi$ is a closed bounded subset of the $\mu$-complete space $G$. Therefore the set $\Phi$ is compact. The proof is complete.

\section{Relations between linear equivalent spaces}

Let $R$ be a topological semiring and $E, F$ be non-trivial locally bounded topological $R$-modules. The $R$-module $E \times F$ is locally bounded. We identify $E$ with the $R$-submodule $E \times\{0\}$ of $E \times F$ and $F$ with the $R$-submodule $\{0\} \times F$ of $E \times F$.

Fix two non-empty $R$-Tychonoff spaces $X$ and $Y$ with the properties:

- for any non-bounded subset $L$ of $X$ there exists $f \in C(X, E)$ such that the set $f(L)$ is not $a$-bounded in $E$;

- for any non-bounded subset $L$ of $Y$ there exists $f \in C(Y, F)$ such that the set $f(L)$ is not $a$-bounded in $F$.

Fix now a continuous linear homeomorphism $u: C_{p}(X, E) \longrightarrow C_{p}(Y, F)$. Then the mapping $v: M_{p}(Y, F, E \times F) \longrightarrow M_{p}(X, E, E \times F)$, where $v(\eta)=\eta \circ u$ for each $\eta \in M_{p}(Y, F, E \times F)$, is a linear homeomorphism.

For each $x \in X$ and each $f \in C_{p}(X, E)$ we put $\varepsilon_{x}(f)=\left(\xi_{x}(f), 0\right)=(f(x), 0) \in$ $E \subseteq E \times F$. For each $y \in Y$ and each $g \in C_{p}(Y, F)$ we put $\delta_{y}(g)=\left(0, \xi_{y}(g)\right)$ $=(0, g(y)) \in F \subseteq E \times F$. Realy, we can assume that $\varepsilon_{x}=\xi_{x}$ and $\delta_{y}=\xi_{y}$. Obviously, $v^{-1}\left(\varepsilon_{x}\right)=\varepsilon_{x} \circ u^{-1} \in M_{p}(Y, F, E \times F) \backslash\{0\}$ and $v\left(\delta_{y}\right)=\delta_{y} \circ u \in$ $M_{p}(X, E, E \times F) \backslash\{0\}$. Hence, for each $x \in X$ and each $\left.y \in Y\right)$ we can put $\varphi(x)=\operatorname{supp}_{Y}\left(v^{-1}\left(\varepsilon_{x}\right)\right)$ and $\psi(y)=\operatorname{supp}_{X}\left(v\left(\delta_{y}\right)\right)$.

Property 7.1. $\varphi: X \rightarrow Y$ and $\psi: Y \rightarrow X$ are l.s.c. set-valued mappings and $\varphi(x), \psi(y)$ are finite non-empty sets for all points $x \in X$ and $y \in Y$.

Proof. Follows from Proposition 4.1 and Theorem 5.1.

Property 7.2. Let $y_{0} \in Y, f \in C(X, E)$ and $f\left(\psi\left(y_{0}\right)\right)=0$. Then $u(f)\left(y_{0}\right)=0$.

Proof. For any $\eta \in M_{p}(Y, F, E \times F)$ and $g \in C(X, E)$ we have $v(\eta)(g)=\eta(u(g))$ $(\eta \circ u)(g)$. Since $f\left(\operatorname{supp}_{X}\left(v\left(\delta_{y_{0}}\right)\right)\right)=f\left(\psi\left(y_{0}\right)\right)=0$, we have $\left(\delta_{y_{0}} \circ u\right)(f)=0$ and $u(f)\left(y_{0}\right)=\delta_{y_{0}}(u(f))=\left(\delta_{y_{0}} \circ u\right)(f)=0$. The proof is complete.

5.1. Corollary. If $f, g \in C(X, E)$ and $f|\psi(y)=g| \psi(y)$, then $u(f)(y)=u(g)(y)$.

Property 7.3. $x \in \psi(\varphi(x))$ for every point $x \in X$ and $y \in \varphi(\psi(y))$ for every point $y \in Y$.

Proof. For every $x \in X$ the sets $\varphi(x)$ and $\psi(\varphi(x))$ are finite and closed. Assume that $x_{0} \in X$ and $x_{0} \notin \psi\left(\varphi\left(x_{0}\right)\right)=H$. Fix $f \in C(X, E)$ such that $f\left(x_{0}\right)=b \neq 0$ and $f(H)=f\left(\psi\left(\varphi\left(x_{0}\right)\right)\right)=0$. Since $\psi(y) \subseteq H$ and $f(H)=0$ for any $y \in \varphi\left(x_{0}\right)$ by virtue of Property 7.2, we have $u(f)(y)=0$ for each $y \in \varphi\left(x_{0}\right)$. Since $u(f)(y)=0$ for each $y \in \varphi\left(x_{0}\right)$, by virtue of Property 7.2, we have $f\left(x_{0}\right)=u^{-1}(u(f))\left(x_{0}\right)=0$. By construction, we have $f\left(x_{0}\right) \neq 0$, a contradiction.

Property 7.4. If $H$ is dense subset of $Y$, then $\psi(H)$ is a dense subset of $X$ provided $u$ is an injection. 
Proof. Assume that $x_{0} \notin c l_{X} \psi(H)$. Then there exists $f \in C(X, E)$ such that $f\left(x_{0}\right) \neq 0$ and $f(\psi(H))=0$. Since $f(\psi(H))=0$ for any $y \in Y$, by virtue of Property 7.2 , we have $u(f)(y)=0$ for any $y \in Y$. Thus $u(f)=0$. Hence $f=0$, a contradiction.

From the above properties follows

5.2. Corollary. The space $X$ is separable if and only if the space $Y$ is separable. In general, $d(X)=d(Y)$.

Property 7.5. $\varphi(H)$ is a bounded set of $Y$ for each bounded set $H$ of $X$.

Proof. Let $H$ be a bounded subset of $X$. Then $H$ is a bounded subset of $M_{p}(X, E, E \times$ $F)$ and respectively $v^{-1}(H)$ is a bounded subset of $M_{p}(Y, F, E \times F)$. By Theorem 4.4 the set $\operatorname{supp}_{Y}\left(v^{-1}(H)\right)$ is a bounded subset of $Y$. The proof is complete.

Property 7.6. Let $E$ and $F$ be metrizable spaces, $X$ be a compactly E-full space and $Y$ be a compactly $F$-full space. Then the space $X$ is $\mu$-complete if and only if the space $Y$ is $\mu$-complete.

Proof. Let $X$ be a $\mu$-complete space. Then $M_{p}(X, E, E \times F)$ and $M_{p}(Y, F, E \times F)$, by virtue of Theorem 4.10, are $\mu$-complete spaces. By Theorem 4.10 the space $Y$ is $\mu$-complete too. The proof is complete.

As in [3] we say that the pair of set-valued mappings $\theta: X \longrightarrow Y$ and $\pi: Y \longrightarrow$ $X$ is called lower-reflective if it has the following conditions:

1l. $\theta$ and $\pi$ are l.s.c.

2l. $\theta(x)$ and $\pi(x)$ are finite sets for all points $x \in X$ and $y \in Y$.

3l. $x \in \pi(\theta(x))$ and $y \in \theta(\pi(y))$ for all points $x \in X$ and $y \in Y$.

Also, as in [3] we say that the pair of set-valued mappings $\theta: X \longrightarrow Y$ and $\pi: Y \longrightarrow X$ is called upper-reflective if it has the following conditions:

1u. $\theta(F)$ is a bounded subset of $Y$ for each bounded subset $F$ of $X$.

$2 u$. $\pi(\Phi)$ is a bounded subset of $X$ for each bounded subset $\Phi$ of $Y$.

3u. $x \in c l_{X} \pi(\theta(x))$ and $y \in c l_{Y} \theta(\pi(y))$ for all points $x \in X$ and $y \in Y$.

General conclusion: The set valued mappings $\varphi: X \longrightarrow Y$ and $\psi: Y \longrightarrow X$ forms an equivalence of $X$ and $Y$ in sense of article [3]. Thus the general theorems from [3] can be extended for the mappings in topological $R$-modules. In the following sections we formulate the general theorems for the $R$-modules, where $R$ is a topological semiring.

\section{Application to perfect properties}

We say that the property $\mathcal{P}$ is a perfect property if for any continuous perfect mapping $f: X \longrightarrow Y$ of $X$ onto $Y$ we have $X \in \mathcal{P}$ if and only if $Y \in \mathcal{P}$. We say that the property $\mathcal{P}$ is a strongly perfect property if it is perfect and any space with property $\mathcal{P}$ is $\mu$-complete.

6.1. Example. From the Example $6.2[3]$ the following properties are perfect: to be a compact space; to be a paracompact $p$-space; to be a paracompact space; to be a metacompact space; to be a $k$-scattered space; to be a monotonically $p$-space; 
to be a monotonically Čech complete space; to be a Čech complete space; to be a Lindelöf space; to be a Lindelöf $\Sigma$-space; to be a subparacompact space; to be a locally compact space.

6.2. Example. The following properties are strongly perfect: to be a compact space; to be a paracompact $p$-space; to be a paracompact space; to be a $\mu$-complete metacompact space; to be a $k$-scattered $\mu$-complete space; to be a $\mu$-complete monotonically $p$-space; to be a $\mu$-complete monotonically Čech complete space; to be a $\mu$-complete Čech complete space; to be a Lindelöf space; to be a Lindelöf $\Sigma$-space; to be a $\mu$-complete subparacompact space; to be a $\mu$-complete locally compact space.

A space $X$ is called a $w q$-space if for any point $x \in X$ there exists a sequence $\left\{U_{n}: n \in \mathbb{N}\right\}$ of open subsets of $X$ such that $x \in \cap\left\{U_{n}: n \in \mathbb{N}\right\}$ and each set $\left\{x_{n} \in U_{n}: n \in \mathbb{N}\right\}$ is bounded in $X$.

A space $X$ is pseudocompact if the set $X$ is bounded in the space $X$. Any pseudocompact space is a $w q$-space.

6.3. Theorem. Let $R$ be a topological semiring and $E$ and $F$ be non-trivial locally bounded topological $R$-modules. Fix two non-empty $R$-Tychonoff spaces $X$ and $Y$ with the properties:

- for any non-bounded subset $L$ of $X$ there exists $f \in C(X, E)$ such that the set $f(L)$ is not $a$-bounded in $E$;

- for any non-bounded subset $L$ of $Y$ there exists $f \in C(Y, F)$ such that the set $f(L)$ is not $a$-bounded in $F$.

Assume that $u: C_{p}(X, E) \longrightarrow C_{p}(Y, F)$ is a linear homeomorphism. Then:

1. $X$ is a pseudocompact space if and only if $Y$ is a pseudocompact space.

2. If $\mathcal{P}$ is a perfect property and $X, Y$ are $\mu$-complete $w q$-spaces, then $X \in \mathcal{P}$ if and only if $Y \in \mathcal{P}$.

Proof. Consider the set-valued mappings $\varphi: X \longrightarrow Y$ and $\psi: Y \longrightarrow X$ constructed in the Section 7.

Let $X$ be a pseudocompact space. Then $X$ is a bounded subset of the space $X$. Hence $Y=\varphi(X)$ is a bounded subset of $Y$ and $Y$ is a pseudocompact space. Assertion 1 is proved.

Assume that $\mathcal{P}$ is a perfect property and $X, Y$ are $\mu$-complete $w q$-spaces. Suppose that $X \in \mathcal{P}$. By virtue of Theorem 2.5 from [3], there exist a space $Z$ and two perfect single-valued mappings $f: Z \longrightarrow X$ and $g: Z \longrightarrow Y$ onto $X$ and $Y$, respectively. Hence, $Y, Z \in \mathcal{P}$. Assertion 2 is proved. The proof is complete.

6.4. Theorem. Let $R$ be a topological semiring and $E$ and $F$ be non-trivial metrizable locally bounded topological $R$-modules. Fix two non-empty spaces $X$ and $Y$ with the properties:

- $X$ is an $R$-Tychonoff compactly $E$-full space and for any non-bounded subset $L$ of $X$ there exists $f \in C(X, E)$ such that the set $f(L)$ is not $a$-bounded in $E$;

- $Y$ is an $R$-Tychonoff compactly $E$-full space and for any non-bounded subset $L$ of $Y$ there exists $f \in C(Y, F)$ such that the set $f(L)$ is not $a$-bounded in $F$.

Assume that $u: C_{p}(X, E) \longrightarrow C_{p}(Y, F)$ is a linear homeomorphism. Then:

1. The space $X$ is $\mu$-complete if and only if the space $Y$ is $\mu$-complete. 
2. $X$ is a compact space if and only if $Y$ is a compact space.

3 . If $\mathcal{P}$ is a strongly perfect property and $X, Y$ are $w q$-spaces, then $X \in \mathcal{P}$ if and only if $Y \in \mathcal{P}$.

Proof. Consider the set-valued mappings $\varphi: X \longrightarrow Y$ and $\psi: Y \longrightarrow X$ constructed in the Section 7. Assertion 1 follows from Property 7.7.

Assume that $\mathcal{P}$ is a strongly perfect property and $X, Y$ are $w q$-spaces. Suppose that $X \in \mathcal{P}$. By definition of a strongly perfect property, $X$ is a $\mu$-complete space. From assertion 1 it follows that $Y$ is a $\mu$-complete space too. By virtue of Theorem 2.5 from [3], there exist a space $Z$ and two perfect single-valued mappings $f: Z \longrightarrow X$ and $g: Z \longrightarrow Y$ onto $X$ and $Y$, respectively. Hence, $Y, Z \in \mathcal{P}$. Assertion 3 is proved.

Let $X$ be a compact space. By virtue of Theorem $6.3, Y$ is a pseudocompact space. Hence $X$ and $Y$ are $w q$-spaces. Assertion 3 completes proof of Assertion 2. The proof is complete.

\section{Application to open properties}

We say that the property $\mathcal{P}$ is an $o f$-property (open finite property) if for any continuous open finite-to-one mapping $f: X \longrightarrow Y$ and any subspace $Z$ of $X$ we have $Z \in \mathcal{P}$ if and only if $f(Z) \in \mathcal{P}$ (see [3]).

7.1. Example. From the results from [3] and [5] the following properties are ofproperties: to be hereditarily Lindelöf; to be $\sigma$-space; to be hereditarily separable; to be $\sigma$-metrizable; to be $\sigma$-scattered; to be $\sigma$-discrete space.

7.2. Example. Let $\tau$ be an infinite cardinal. Consider the properties: $X \in e(\tau)$ if and only if $e(X) \leq \tau ; X \in d(\tau)$ if and only if $d(X) \leq \tau ; X \in h d(\tau)$ if and only if $h d(X) \leq \tau ; X \in h l(\tau)$ if and only if $h l(X) \leq \tau$.

Then $e(\tau), d(\tau), h d(\tau), h l(\tau)$ are $o f$-properties.

7.3. Theorem. Let $R$ be a topological semiring and $E, F$ be non-trivial locally bounded topological $R$-modules. Fix two non-empty $R$-Tychonoff spaces $X$ and $Y$ with the properties:

- for any non-bounded subset $L$ of $X$ there exists $f \in C(X, E)$ such that the set $f(L)$ is not $a$-bounded in $E$;

- for any non-bounded subset $L$ of $Y$ there exists $f \in C(Y, F)$ such that the set $f(L)$ is not $a$-bounded in $F$.

Assume that $u: C_{p}(X, E) \longrightarrow C_{p}(Y, F)$ is a linear homeomorphism. If $\mathcal{P}$ is an $o f$-property, then $X \in \mathcal{P}$ if and only if $Y \in \mathcal{P}$.

Proof. Consider the set-valued mappings $\varphi: X \longrightarrow Y$ and $\psi: Y \longrightarrow X$ constructed in the Section 7. As in [3] (see Theorem 2.1 from [3]) we put $Z=$ $\cup\{\{x\} \times \varphi(x): x \in X\}$ and $S=\cup\{\psi(y) \times\{y\}: y \in Y\}$ as subspaces of the spaces $X \times Y, f(x, y)=x$ and $g(x, y)=y$ for any point $(x, y) \in X \times Y$. Then $f: Z \longrightarrow X$ and $g: S \longrightarrow Y$ are continuous open finite-to-one mappings. If $D=Z \cap S$, then from Property 7.4 it follows that $f(D)=X$ and $g(D)=Y$. Hence $X \in \mathcal{P}$ if and only if $Y \in \mathcal{P}$. The proof is complete. 


\section{8. $l_{p}(E, F)$-equivalence and metrizability}

8.1. Theorem. Let $R$ be a topological semiring and $E, F$ be non-trivial metrizable locally bounded topological $R$-modules. Fix two non-empty spaces $X$ and $Y$ with the properties:

- $X$ is an $R$-Tychonoff compactly $E$-full space and for any non-bounded subset $L$ of $X$ there exists $f \in C(X, E)$ such that the set $f(L)$ is not $a$-bounded in $E$;

- $Y$ is an $R$-Tychonoff compactly $E$-full space and for any non-bounded subset $L$ of $Y$ there exists $f \in C(Y, F)$ such that the set $f(L)$ is not $a$-bounded in $F$.

Let $X$ and $Y$ be $l_{p}(E)$-equivalent spaces. Then:

1. $X$ is a compact metrizable space if and only if $Y$ is a compact metrizable space.

2. If $X$ is a metrizable space, then the space $Y$ is metrizable if and only if $Y$ is a $w q$-space.

Proof. Any metrizable space is a $w q$-space.

Let $X$ be a metrizable space and $Y$ be a $w q$-space. Since $X$ is metrizable, by virtue of Theorem 6.3, $Y$ is a paracompact $p$-space. From Theorem 7.3 it follows that $Y$ is a $\sigma$-space. If a paracompact space $Y$ is a $\sigma$-space and a $p$-space, then $Y$ is metrizable [13]. Assertion 2 is proved.

Assertion 1 follows from the Assertion 2 and Theorem 6.3. The proof is complete.

\section{References}

[1] A. V. Arhangel'skii, Topological Function Spaces, Mathematics and its Applications (Soviet Series), vol. 78, Kluwer Academic Publishers Group, Dordrecht, 1992.

[2] A. V. Arhangel'skii, On linear homomorphisms of function spaces, Doklady Acad. Nauk SSSR 264 (1982), no. 6, 1289-1292. English translation: Soviet Math. Dokl. 25 (1982), $852-855$.

[3] M.M. Choban, General theorems on functional equivalence of topological spaces, Topology Appl. 89 (1998), 223-239.

[4] M.M. Choban, Algebraical equivalence of topological spaces, Buletinul Acad. de Ştiinţe a Republicii Moldova, Matematica, 1 (2001), 12-36.

[5] M. Choban, Open finite-to-one mappings, Soviet Math. Dokl. 8 (1967), 603-603.

[6] M.M. Choban, On the theory of topological algebraic systems, Trudy Moskovskogo Matem. Obshchestva 48 (1985), 106-149. English translation: Trans. Moscow Math. Soc. 48, 1986, 115-159.

[7] M.M. Choban, Some topics in topological algebra, Topology Appl. 54 (1993), 183-202.

[8] M.M. Choban, R. N. Dumbrăveanu, $l_{p}(R)$-equivalence of topological spaces and topological modules, Buletinul Academiei de Stiinte a Rep. Moldova, Matematica 1 (2015), 20-47.

[9] R. Engelking, General Topology, PWN, Warsawa 1977.

[10] J. S. Golan, Semirings and their applications, Springer, 1999.

[11] V. P. Maslov, Idempotent analysis, American Mathematical Society, 1992.

[12] J. van Mill, The infinite-dimensional topology of function spaces, North-Holland Mathematical Library, Amsterdam, vol. 64, 2001.

[13] A. Okuyama, A survey of the theory of $\sigma$-spaces, General Topology Appl. 1 (1971), 57-63.

[14] V. Valov, Function spaces, Topology Appl. 81 (1997), no. 1, 1-22.

[15] S. Warner, Topological rings, North-Holland mathematics studies. Elsevier, v. 178, 1993. 